\title{
Rheological and dynamic-mechanical behavior of carbon nanotube/vinyl ester-polyester suspensions and their nanocomposites
}

\author{
A.T. Seyhan ${ }^{\text {a }}$, F.H. Gojny ${ }^{b}$, M. Tanoğlu ${ }^{a, *}$, K. Schulte ${ }^{b}$ \\ ${ }^{a}$ Izmir Institute of Technology (IZTECH), Mechanical Engineering Department, 35430 Izmir, Turkey \\ b Polymer Composites, Technical University Hamburg-Harburg (TUHH), Denickestrasse 15, D-21073 Hamburg, Germany
}

Received 10 December 2006; received in revised form 16 March 2007; accepted 10 April 2007

Available online 1 May 2007

\begin{abstract}
Rheological properties of vinyl ester-polyester resin suspensions containing various amounts $(0.05,0.1$ and 0.3 wt. $\%)$ of multi walled carbon nanotubes (MWCNT) with and without amine functional groups $\left(-\mathrm{NH}_{2}\right)$ were investigated by utilization of oscillatory rheometer with parallel plate geometry. Dispersion of corresponding carbon nanotubes within the resin blend was accomplished employing high shear mixing technique (3-roll milling). Based on the dynamic viscoelastic measurements, it was observed that at $0.3 \mathrm{wt} . \%$ of CNT loadings, storage modulus $\left(G^{\prime}\right)$ values of suspensions containing MWCNTs and MWCNT- $\mathrm{NH}_{2}$ exhibited frequency-independent pseudo solid like behavior especially at lower frequencies. Moreover, the loss modulus $\left(G^{\prime \prime}\right)$ values of the resin suspensions with respect to frequency were observed to increase with an increase in contents of CNTs within the resin blend. In addition, steady shear viscosity measurements implied that at each given loading rate, the resin suspensions demonstrated shear thinning behavior regardless of amine functional groups, while the neat resin blend was almost the Newtonian fluid. Furthermore, dynamic mechanical behavior of the nanocomposites achieved by polymerizing the resin blend suspensions with MWCNTs and MWCNT- $\mathrm{NH}_{2}$ was investigated through dynamic mechanical thermal analyzer (DMTA). It was revealed that storage modulus $\left(E^{\prime}\right)$ and the loss modulus $\left(E^{\prime \prime}\right)$ values of the resulting nanocomposites increased with regard to carbon nanotubes incorporated into the resin blend. In addition, at each given loading rate, nanocomposites containing MWCNT- $\mathrm{NH}_{2}$ possessed larger loss and storage modulus values as well as higher glass transition temperatures $\left(T_{\mathrm{g}}\right)$ as compared to those with MWCNTs. These findings were attributed to evidences for contribution of amine functional groups to chemical interactions at the interface between CNTs and the resin blend matrix. Transmission electron microscopy (TEM) studies performed on the cured resin samples approved that the dispersion state of carbon nanotubes with and without amine functional groups within the matrix resin blend was adequate. This implies that 3-roll milling process described herein is very appropriate technique for blending of carbon nanotubes with a liquid thermoset resin to manufacture nanocomposites with enhanced final properties.
\end{abstract}

(c) 2007 Elsevier Ltd. All rights reserved.

Keywords: Carbon nanotubes; Thermosets; Rheology; Nanocomposites; Thermal properties; TEM

\footnotetext{
${ }^{*}$ Corresponding author. Tel.: +90 232750 7806/7890.

E-mail address: metintanoglu@iyte.edu.tr (M. Tanoğlu).
} 


\section{Introduction}

Addition of nano-scale particles into polymers has been the recent study of interest in materials science to develop functional nanocomposites offering enhanced properties as compared to conventional polymer based composites that contains micro-scale particulates such as carbon black (CB) or glass micro spheres [1-3]. Due to their high aspect ratio as well as excellent mechanical, thermal and electrical properties, CNTs have been recently considered as an ideal nano-filler to fabricate various types of polymer based nanocomposites [1-4]. However, the poor dispersion states of CNTs within the surrounding resin matrix due to their huge surface area and inert surfaces leads to some limitations for handling and processing [1-5]. Therefore, achievement of homogeneous dispersion of CNTs within polymers is the key for the realization of the desired enhancement in the final properties of nanocomposites. The degree of dispersion is commonly regarded as functions of the size of the dispersed particles, wettability by disperse medium, and nature of the attractive forces between the corresponding constituents [7]. From that point of view, some physical and chemical surface treatments have been applied for CNTs to improve their dispersion state and compatibility with the surrounding matrix resin [2-4]. Direct mixing and sonication have been the most common techniques to disperse CNTs within thermoset polymer resins such as epoxy, polyester or vinyl ester $[2,3]$. However, these methods were found to be ineffective to prevent the agglomerates of CNTs [1-7]. Shear intensive 3-roll milling process has been recently applied for the same purpose. Gojny et al. [4] revealed that double walled carbon nanotubes (DWCNTs) exhibited adequate dispersion within the epoxy resins through 3-roll milling technique, based upon transmission electron microscopy (TEM) investigations. In the same study, they also showed that blending of DWCNTs with very low content $(0.1 \mathrm{wt} . \%)$ of epoxy resin via 3-roll milling process improved the mechanical properties such as Young's modulus and strain to failure ratio of the corresponding epoxy resins.

In addition to microscopic techniques such as TEM, rheological examination of polymer suspensions containing CNTs has been also recently performed to investigate the dispersion state of CNTs in the corresponding resin media [7-9]. It was revealed that the Brownian motion of CNT particles with huge aspect ratio results in more considerable viscoelastic rheological behavior as compared to those of micrometer size short fiber or particles such as carbon black $[7,8]$. However, the number of works reported in the literature on the rheological behavior of CNT/polymer systems is quite limited [6-11]. The rheological behavior of concentrated aqueous nanotube dispersions was studied by Kinloch et al. [7]. They found that the dispersion state of MWCNTs is highly sensitive to the applied strain in the linear viscoelastic region and the storage and loss modulus were independent of frequency. Mitchell et al. [10] investigated the linear viscoelastic properties of polystyrene (PS) nanocomposites containing SWCNTs with and without surface functional groups. They found that the nanotubes with functional groups have better dispersion in PS than those without any functional group. The authors also revealed that nanocomposites with functionalized nanotubes gave higher storage modulus and complex viscosity values at low frequency level. Kim et al. [8] studied the rheological behavior of epoxy resin suspensions containing amine, acid and plasma treated carbon nanotubes. It was found that the surface modified CNT/epoxy suspensions exhibited a very strong shear thinning behavior and higher shear viscosity than those with untreated CNTs due to the enhanced interfacial bonding between CNTs and the corresponding epoxy resin. Song and Youn [9] also performed a similar study and claimed that poorly dispersed CNTs within epoxy resin leads to higher storage, loss modulus and complex viscosity in the resulting resin suspensions compared to homogenously dispersed CNTs.

Measuring the temperature dependent material properties is also critical for determination of appropriate processing conditions to predict the behavior of polymeric components during their service life [2-12]. The dispersion state of carbon nanotubes influences the dynamic mechanical properties of the resulting nanocomposites. Fidelus et al. [12] investigated the dynamic mechanical properties of the epoxy based nanocomposites containing MWCNTs and SWCNTs. They found an 8\% increase of storage modulus in the nanocomposites with SWCNTs relative to the neat epoxy, while those with MWCNTs showed almost no substantial change in the elastic modulus values. They also revealed that glass transition temperature of the nanocomposites containing SWCNTs or MWCNTs increases slightly with respect to nanotube contents.

In this study, the rheological behavior of vinyl ester/polyester based suspensions containing 
MWCNTs and MWCNT-NH $\mathrm{N}_{2}$ was determined measuring dynamic viscoelastic and steady shear properties of the suspensions via parallel plate oscillatory rheometer. Furthermore, the corresponding CNT/polymer suspensions were cross-linked using peroxide (MEKP) initiator. Dynamic mechanical behavior of the resulting nanocomposites was investigated by dynamic mechanical thermal analyzer (DMTA) to relate the influence of dispersion state of CNTs within the blend with final mechanical and thermal properties of the corresponding nanocomposites. Transmission electron microscopy (TEM) was employed to investigate the dispersion state of CNTs within nanocomposites. The effect of the amine functional groups on the surfaces of carbon nanotubes upon their dispersion state and chemical interactions at the interface between CNTs and the resin blend were discussed in details considering the liquid and solid state of each involved resin blend, respectively.

\section{Experimental}

\subsection{Materials}

Thin multiwalled carbon nanotubes (MWCNTs) and amino-functionalized multiwalled carbon nanotubes $\left(\mathrm{MWCNT}-\mathrm{NH}_{2}\right.$ ) were obtained from Nanocyl (Namur Belgium) and used as nanofillers within the corresponding resin blend. CNTs have an average diameter of $15 \mathrm{~nm}$ with a length of approximately $50 \mu \mathrm{m}$. Styrene-free polyester (Poliya 420) and vinyl ester resin (Polives 701, a bisphenol A epoxy based vinyl ester resin with $35 \mathrm{wt} . \%$ of styrene) were obtained from Poliya Polyester, Turkey. Styrene emission agent BKY 740, purchased from Alton Chemie, Germany, was employed to prevent the styrene evaporation from the resin blend during the polymerization reaction. The formulized resin blend was composed of $25 \mathrm{wt} . \%$ of Poliya 420 and $75 \mathrm{wt} . \%$ of Polives 701. BKY 740 was further introduced to the prepared suspension at a ratio of $1 \mathrm{wt} . \%$. To polymerize each corresponding resin blend suspension, Cobalt naphtanate (CoNAP) and methyl ethyl kethone peroxide (MEKP) were introduced to the system as accelerator and initiator, respectively.

\subsection{Three (3)-roll milling process}

Various amounts of MWCNTs and MWCNT$\mathrm{NH}_{2}(0.05,0.1$ and $0.3 \mathrm{wt} . \%)$ were first dispersed in the styrene free (Poliya 420) polyester resin by shear intensive blending via 3-roll milling technique. The collected CNT/polyester resin mixture was subsequently blended with vinyl ester resin at a weight ratio of $(1 / 3)$ by hand mixing for about $10 \mathrm{~min}$ followed by mechanical stirring for about $15 \mathrm{~min}$. After the addition of the styrene emission agent (BKY 740, $1 \mathrm{wt} . \%$ ) into the prepared resin mixture, the CoNAP and MEKP were introduced into the system at a ratio of 0.2 and $1 \mathrm{wt} . \%$, respectively. The resin suspensions were allowed to cure at room temperature followed by post curing at $75^{\circ} \mathrm{C}$ and $120^{\circ} \mathrm{C}$ for $2 \mathrm{~h}$, respectively. Prior to the addition of CoNAP and MEKP into the mixture, liquid samples from each type of the resin suspensions were taken for the rheological characterization. Please note that a special resin blend was used to manufacture nanocomposites because of the fact that some serious problems with commercial unsaturated polyester resin. During 3-roll milling processing, instant styrene evaporation encountered due to heat evolved on the rolls caused increase of the viscosity of resin. Therefore, to overcome these difficulties, the CNTs were first dispersed in specially synthesized high viscous styrene-free polyester resin with the application of high shear forces between gaps of the rolls to break up the agglomerates of the CNTs.

\subsection{Rheological measurements}

A oscillatory rheometer (TA Instruments) with parallel plate geometry (500 micrometer gap, and $50 \mathrm{~mm}$ plate diameter) was used to analyze the vinyl ester/polyester resin blend suspensions with different amounts of carbon nanotubes with and without amine functional groups. Tests were performed in both dynamic and steady modes at room temperature in order to avoid extreme styrene evaporation during the measurements. All measurements were taken in linear viscoelastic region (LVR) in which the storage modulus $\left(G^{\prime}\right)$ and loss modulus $\left(G^{\prime \prime}\right)$ were independent of strain amplitude. Dynamic frequency sweeps (DFS) were then conducted in the LVR to investigate the structure of the suspensions. In the DFS, the strain amplitude was remained constant $35 \%$ through whole frequency range. Please note that prior to main experiments, the corresponding value of strain amplitude was proved to be in the LVR conducting Dynamic Strain Sweeps (DSS) at a constant frequency. During the DFS, the frequency varied stepwise from 0.1 to $80 \mathrm{rad} / \mathrm{s}$. 
Storage modulus $\left(G^{\prime}\right)$ and loss modulus $\left(G^{\prime \prime}\right)$ values were then measured as a function of frequency. Furthermore, steady shear sweeps (SSS) were employed to investigate the flow properties of the suspensions by considering the viscosity as a function of increasing shear rates.

\subsection{Dynamic mechanical characterization of the nanocomposites}

Dynamic mechanical properties of the nanocomposites prepared after curing of each type of the resin suspension were investigated by dynamic mechanical thermal analyzer, (DMTA, GABO EPLOXOR $500 \mathrm{~N}$ ). For the measurements, rectangular specimens of $50 \mathrm{~mm}$ in length, $5 \mathrm{~mm}$ in width and $2 \mathrm{~mm}$ in thickness were sectioned from larger samples. The tests were performed in tensile mode at a frequency of $10 \mathrm{~Hz}$ with a static strain of $0.6 \%$ and dynamic strain of $0.1 \%$, in a temperature range between -50 and $200{ }^{\circ} \mathrm{C}$ with a heating rate of $3{ }^{\circ} \mathrm{C} / \mathrm{min}$. The storage modulus $\left(E^{\prime}\right)$, loss modulus $\left(E^{\prime \prime}\right)$ and the loss tangent $(\tan \delta$ ) were determined as a function of temperature.

\subsection{Transmission electron microscopy}

The transmission electron microscopy (TEM) was conducted to investigate the dispersion state of CNTs with and without amine functional groups within the resulting nanocomposites. The TEM images were taken using a Philips EM 400 at $120 \mathrm{kV}$. Ultra thin films of each type of composites $(50 \mathrm{~nm})$ were obtained by ultra microtome cutting.

\section{Results and discussion}

\subsection{Rheological behavior of the suspensions}

The storage modulus $\left(G^{\prime}\right)$ of the resin suspensions with different concentrations of MWCNT and MWCNT $-\mathrm{NH}_{2}$ is shown in Figs. 1 and 2, respectively. Consequently, storage modulus values were observed to increase with an increase in the oscillatory frequency. Moreover, as the concentrations of the CNTs were increased in the corresponding resin blend, higher storage modulus values were obtained. At $0.3 \mathrm{wt} . \%$ CNT contents, the flow regime of the resin suspensions was significantly altered at low frequencies and a pseudo-solid like behavior was more visible. We referred this as pseudo-solid like behavior since in true-solid like behavior the storage modulus $\left(G^{\prime}\right)$ is fully independent of frequency [11]. In addition, suspensions with 0.1 and $0.3 \mathrm{wt} . \%$ of MWCNTs exhibited slightly higher storage modulus values especially at low frequencies as compared to those with MWCNT- $\mathrm{NH}_{2}$. It was also reported that the CNTs with very small sizes are very likely to produce strong particle-polymer interactions even at very low filling rates

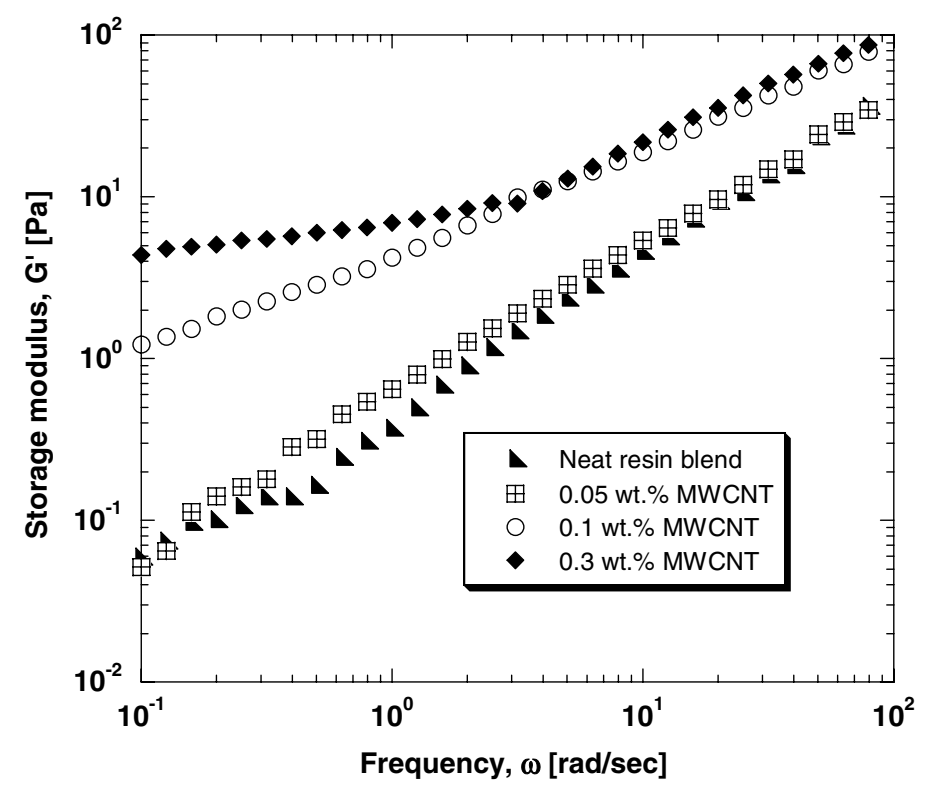

Fig. 1. The frequency dependence of the storage modulus $\left(G^{\prime}\right)$ for the CNT/resin suspensions with various MWCNT contents. 


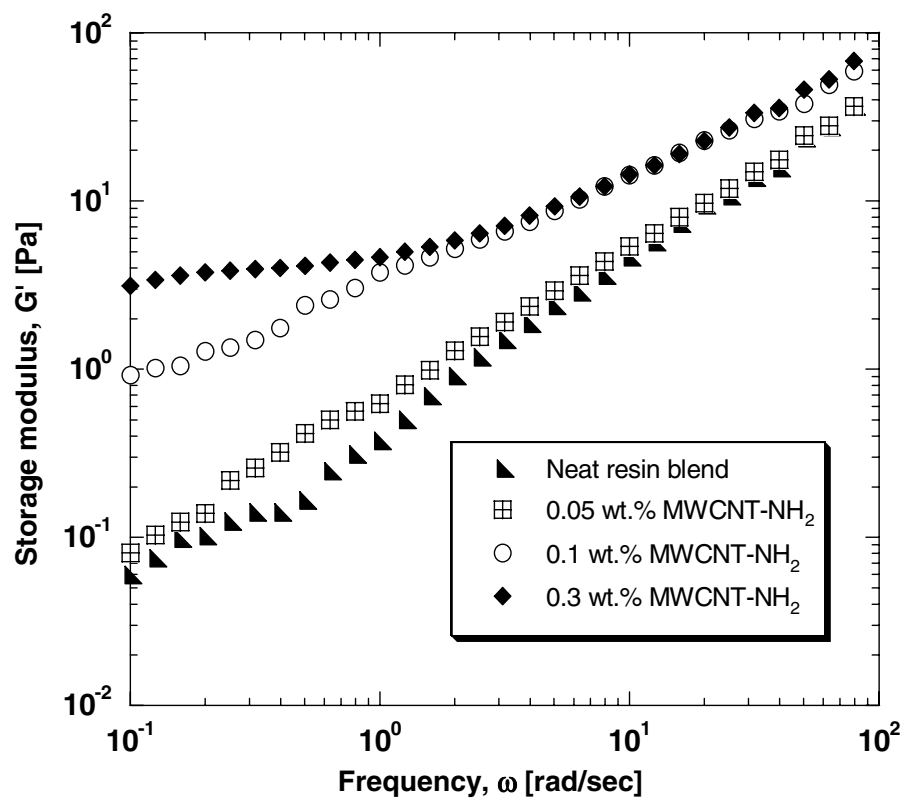

Fig. 2. The frequency dependence of the storage modulus $\left(G^{\prime}\right)$ for the $\mathrm{CNT} /$ resin suspensions with various $\mathrm{MWCNT}-\mathrm{NH}_{2}$ contents.

because of the huge interfacial area between the particles and polymers [8-10]. So, the high aspect ratio and huge surface area of CNTs raise the storage modulus of the resin suspensions. Figs. 3 and 4 show the loss modulus values of polymer suspensions containing MWCNTs and MWCNT- $\mathrm{NH}_{2}$, respectively. It was observed that the loss modulus values as a function of angular frequency increases with the incorporation of carbon nanotubes. In addition, at $0.3 \mathrm{wt} . \%$ loading rate, resin suspension containing untreated CNTs exhibits viscous behavior $\left(G^{\prime \prime}>G^{\prime}\right)$, whereas those with amino functionalized CNTs shows viscoelasticity $\left(G^{\prime \prime} \approx G^{\prime}\right)$. This verifies that amine functional groups over CNT surfaces alter the flow characteristic of resin suspensions as well.

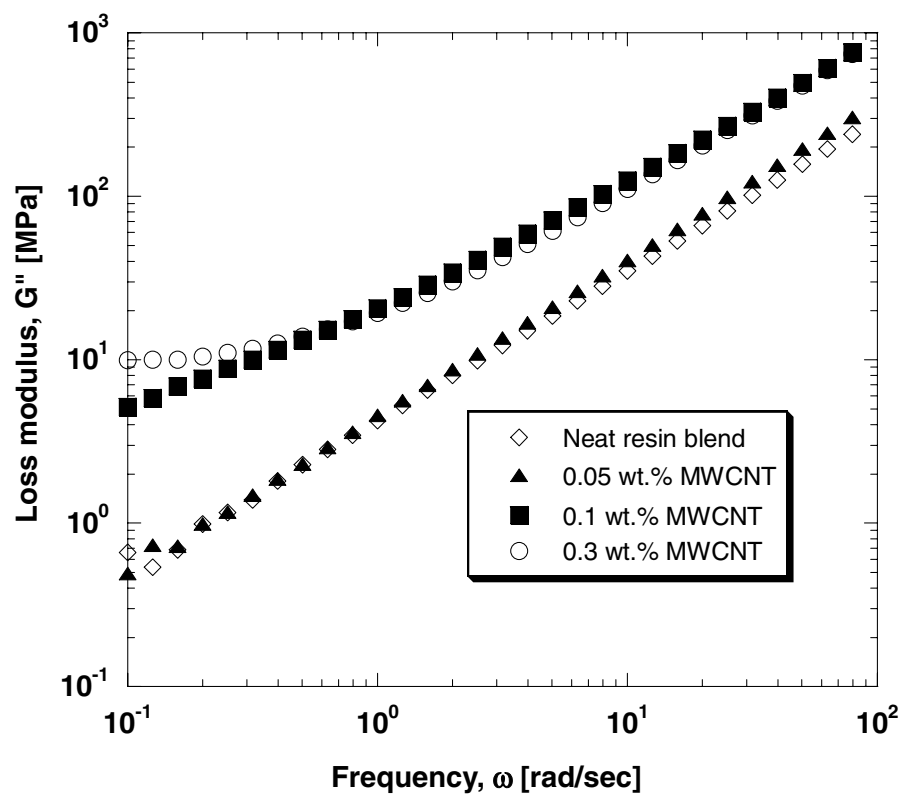

Fig. 3. The frequency dependence of the loss modulus $\left(G^{\prime \prime}\right)$ for the CNT/resin suspensions with various MWCNT contents. 


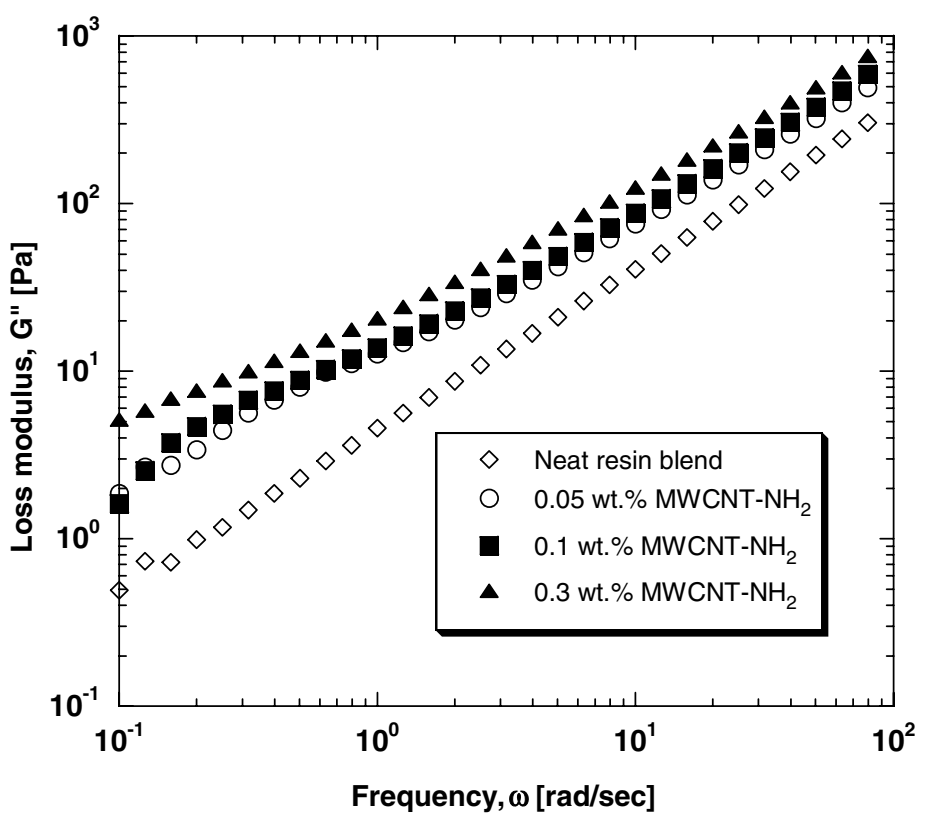

Fig. 4. The frequency dependence of the loss modulus $\left(G^{\prime \prime}\right)$ for the CNT/resin suspensions with various $\mathrm{MWCNT}^{-\mathrm{NH}_{2}}$ contents.

Figs. 5 and 6 give shear viscosity as a function of shear rate for the neat resin blend and resin suspensions containing MWCNTs and MWCNT- $\mathrm{NH}_{2}$, respectively. In principle, shear viscosity of a pure polymer is divided into two distinct regions including the Newtonian and shear thinning regions [9-11]. At low shear rates, the Newtonian region independent of shear rate is observed followed by shear thinning region through which shear viscosity linearly declines with shear rate. As seen in the figures, shear thinning behavior was observed for each resin suspensions with nanotubes such that the viscosity is reduced with an increase in shear rates. Moreover, the neat resin blend showed almost the Newtonian fluid behavior. In other words, the Newtonian region disappeared even if very low content

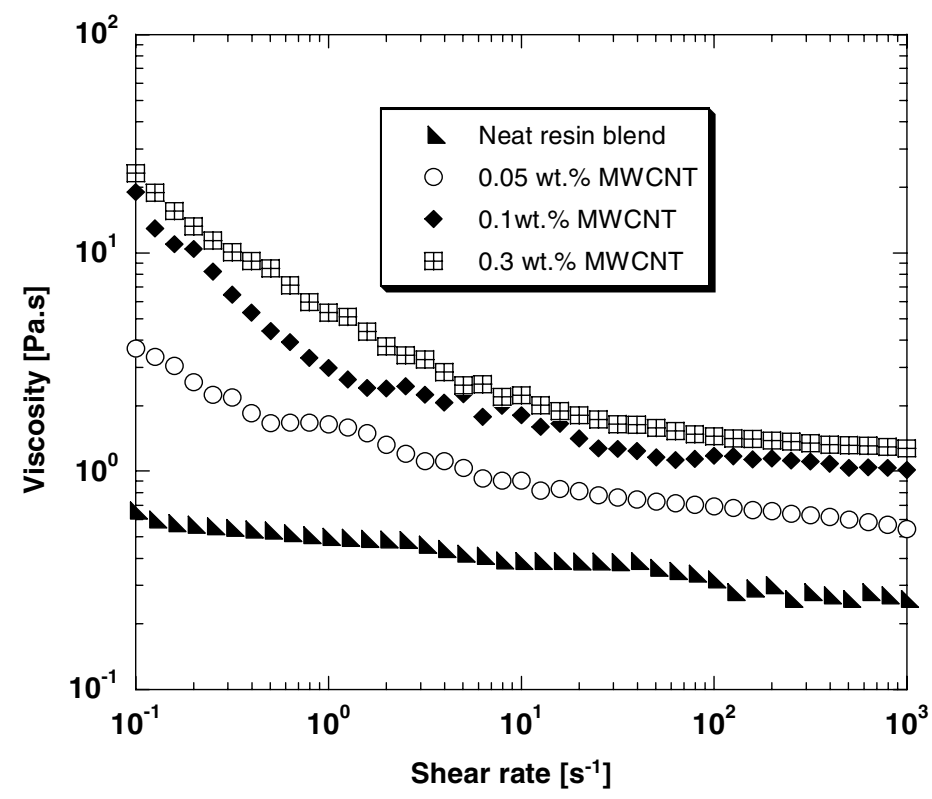

Fig. 5. Shear rate dependency of viscosity of neat resin blend and CNT/resin suspensions with MWCNTs. 


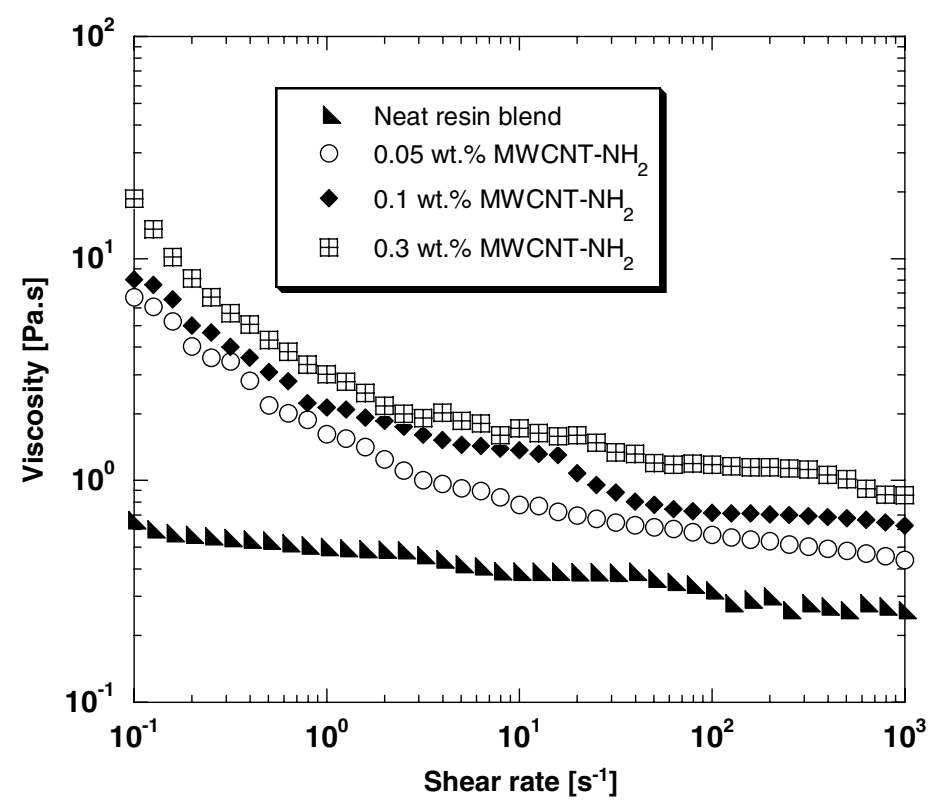

Fig. 6. Shear rate dependency of viscosity of resin blend and CNT/resin suspensions with MWCNT-NH ${ }_{2}$.

of CNTs (0.05 wt.\%) is added into the resin blend. This implies that strong particle-particle interaction of CNTs is one major factor that leads to an increase in shear viscosity of the corresponding suspensions. On the other hand, at $0.1 \mathrm{wt} . \%$ loading rate, the initial shear viscosity of the resin suspension with amino-functionalized nanotubes were slightly lower than those of the suspensions with non-functionalized nanotubes. Moreover, resin suspensions containing $0.3 \mathrm{wt} . \%$ of MWCNTs and MWCNT $-\mathrm{NH}_{2}$ exhibited similar initial viscosity values. So, based on the experimental findings, it was observed that amine functional groups do not significantly contribute to enhancement of dispersion state of CNTs within the corresponding resin blends. In fact, it is not easy to claim a single and precise comment on rheological behavior of polymeric suspensions because it is extremely depend on dispersing state of fillers inside, particle-particle interactions, and interaction between particles and disperse medium (polymer). In the present case, since CNTs have high aspect ratio, surface area and amine functional groups over their surfaces, both particle-polymer resin blend and particle-particle interactions became more crucial, as elucidated in details during analysis of rheological data above. Please note that the amino-functionalized nanotubes used in our experiments were processed in ammonia solution via ball milling process, during which CNTs are broken in length and their aspect ratios are partially diminished. According to information provided by the manufacturer of CNTs (Nanocyl, Belgium), the aspect ratio of multi walled nanotubes with amine functional groups is eventually five times lower than those without any surface treatment. From that point of view, the incorporation of amino-functionalized nanotubes (MWCNT$\mathrm{NH}_{2}$ ) with their reduced aspect ratio into the resin system is expected to result in relatively lower storage and loss modulus values in addition to reduced shear viscosity in their corresponding resin suspensions as compared to those with MWCNTs. However, in our case, the effect of the surface functionalization on the rheological behavior is relatively insignificant especially at higher loading rate of CNTs embedded into the resin blend. This implies a conceivable occurrence of chemical interactions between CNTs and disperse medium (resin blend) through the amine functional groups over the CNTs surfaces. So, the effects of interfacial interactions and particle-particle interactions via distinct aspect ratios of CNTs upon the shear viscosity and dynamic rheological properties of the $\mathrm{CNT} /$ resin systems needs further investigations. Therefore, it is interesting to monitor the thermomechanical properties of the resulting nanocomposites (solid state) prepared for each corresponding resin suspensions containing MWCNTs and MWCNT- $-\mathrm{NH}_{2}$ in order to further follow our approach. Furthermore, TEM was also conducted 
to characterize the dispersion state of CNTs with and without amine functional group within the cured resin blends.

\subsection{Thermo-mechanical properties of the resulting nanocomposites}

Figs. 7 and 8 give storage modulus $\left(E^{\prime}\right)$ and loss factor $(\tan \delta)$ values as a function of temperature obtained from dynamic mechanical measurements for the nanocomposites containing MWCNT and MWCNT- $\mathrm{NH}_{2}$, respectively. The addition of nonfunctionalized and amino-functionalized carbon nanotubes into the polymer system has some considerable effects on the storage modulus in both the glassy and the rubbery states, depending on the nanotube contents within the resin blend. This is due to the stiffening effect of CNTs and interfacial interactions along a huge interfacial area between the CNTs and the polymer matrix. Consequently, CNTs reduced the mobility of the surrounding polymer matrix to some extent leading to an increase in the modulus values. This effect is more pronounced in the glass transition region. As mentioned above, amino-functionalized nanotubes have shorter length (lower aspect ratios) as compared to that without surface treatment as a result of the functionalization process (ball milling process). So, one can expect relatively lower elastic modulus values from the nanocomposites containing MWCNT- $\mathrm{NH}_{2}$ as compared to those with MWCNTs. However, we obtained opposing results in the present case. As an example, the storage modulus values at $20^{\circ} \mathrm{C}$ for the nanocomposites containing $0.3 \mathrm{wt} . \%$ of MWCNT- $\mathrm{NH}_{2}$ and MWCNTs were found to be 3170 and $2930 \mathrm{MPa}$, respectively, which are also higher than that of the neat resin blend (2430 MPa). These results revealed that relatively enhanced dispersion state of the MWCNT- $\mathrm{NH}_{2}$ within the matrix compensates the lower aspect ratio of these tubes and provides higher modulus values to their resulting nanocomposites.

In Figs. 9 and 10, the loss modulus ( $\left.E^{\prime \prime}\right)$ values of the nanocomposites containing MWCNTs and MWCNT- $\mathrm{NH}_{2}$ were given as a function of temperature, respectively. It was found that the loss modulus values at the peak points gets higher, as the nanotube contents increases. In addition, nanocomposites containing amino-functionalized nanotubes results in somewhat higher peak values as compared to those with non-functionalized nanotubes. In brief, the loss modulus indicates the energy converted into heat and can thus be used as a measurement of viscous component or unrecoverable oscillation energy dissipated per cycle. From that point of view, we can further conclude that the satisfactorily dispersed nanotubes with and without treatment would assist in dissipating energy under visco-elastic deformation of the surrounding resin blend matrix. Moreover, higher loss modulus values

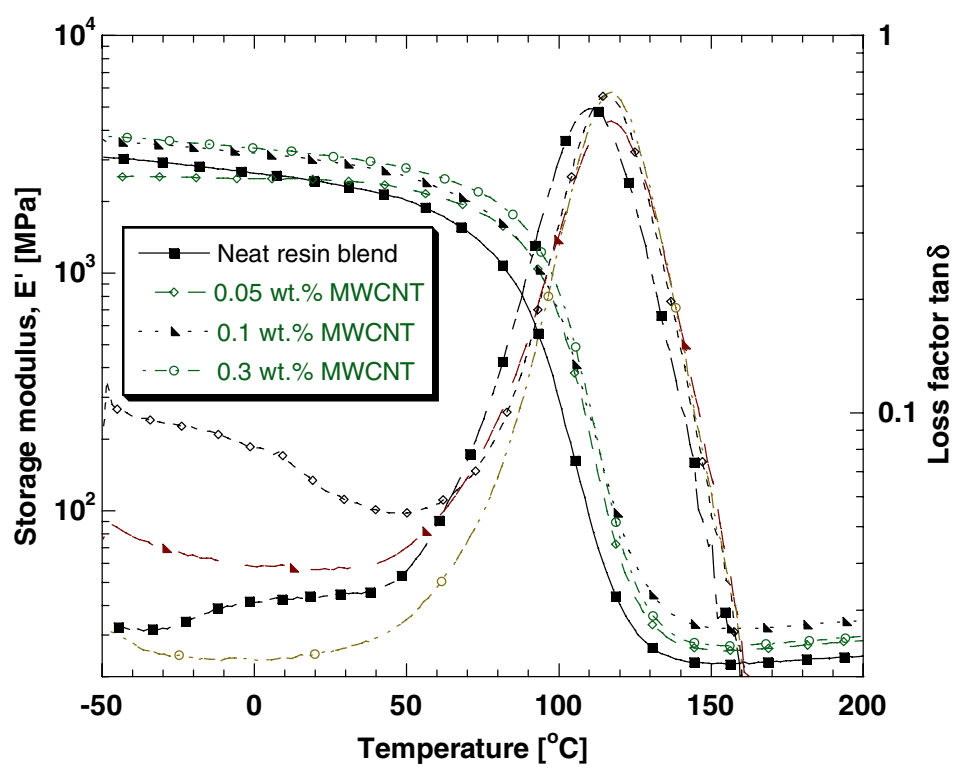

Fig. 7. Storage modulus and loss factor of nanocomposites containing non-functionalized nanotubes (MWCNT). 


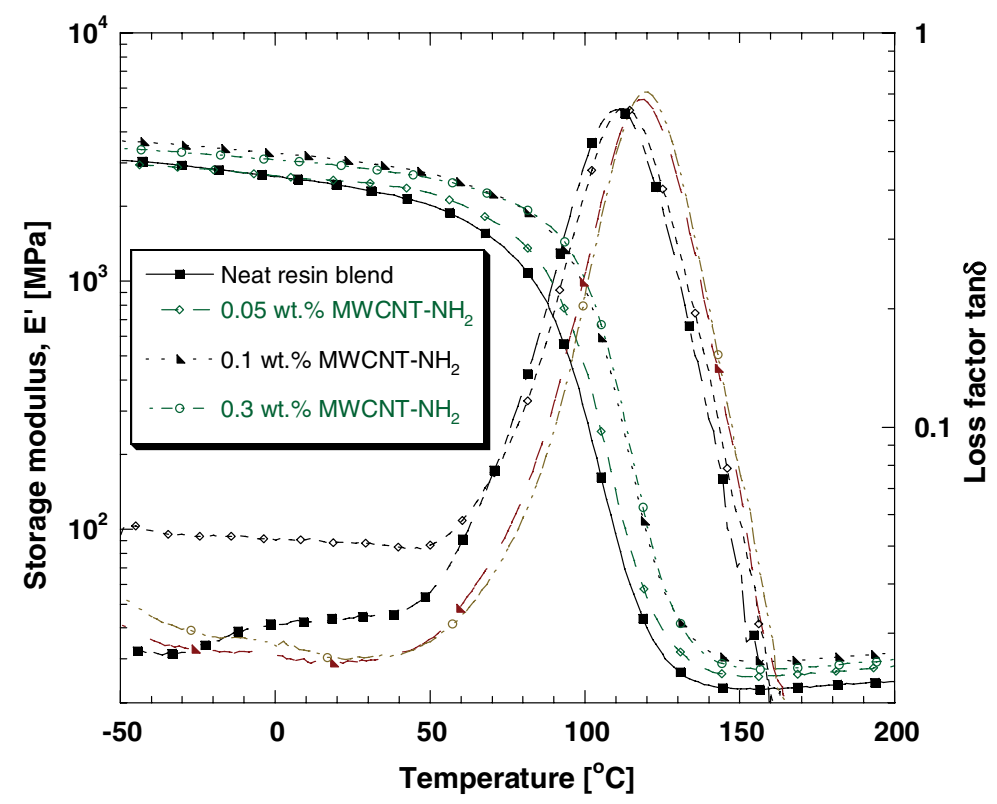

Fig. 8. Storage modulus and loss factor of nanocomposites containing amino-functionalized nanotubes $\left(\mathrm{MWCNT}-\mathrm{NH}_{2}\right)$.

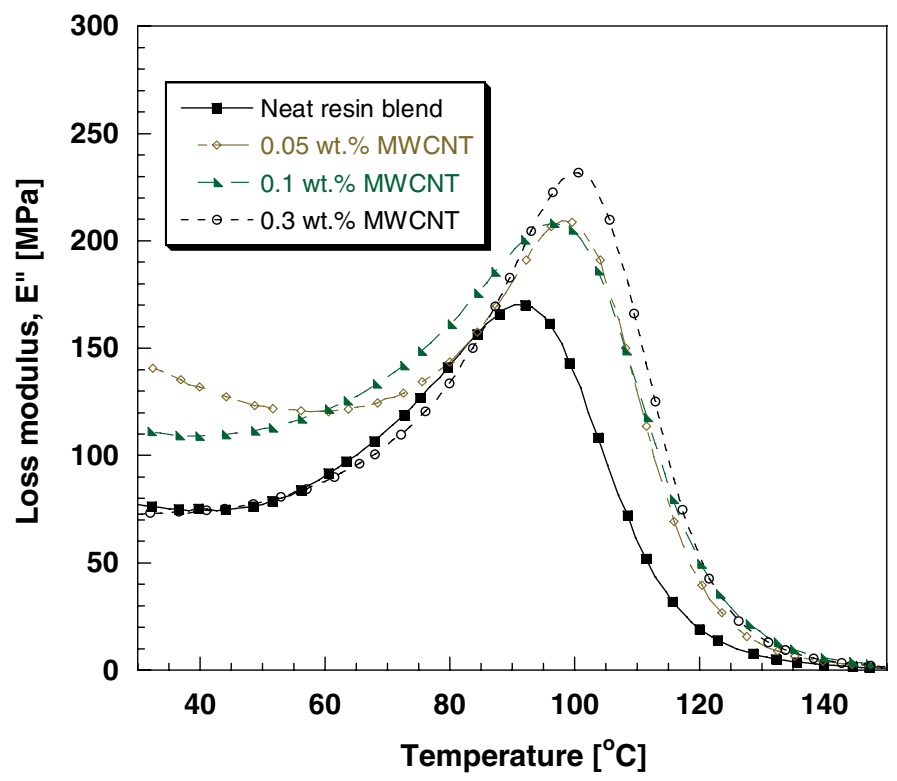

Fig. 9. Loss modulus values of nanocomposites containing non-functionalized nanotubes (MWCNT).

of the nanocomposites with MWCNT- $\mathrm{NH}_{2}$ as compared to those with MWCNTs showed that amine functional groups promotes the involved chemical interactions at the interface in some extend, owing to the same reasons as previously explained.

Fig. 11 shows the glass transition temperature $\left(T_{\mathrm{g}}\right)$ values of the nanocomposites with MWCNT and MWCNT- $\mathrm{NH}_{2}$ obtained from the slope of the storage modulus values in the glass transition zone. As seen in the figure, the addition of nanotubes within the resin blends increased the corresponding $T_{\mathrm{g}}$ values significantly. The mobility of the polymer matrix around the nanotubes is reduced due to the presence of the nanotubes. In fact, interfacial strong bonds are expected to occur between the polymer matrix and the amino-functionalized 


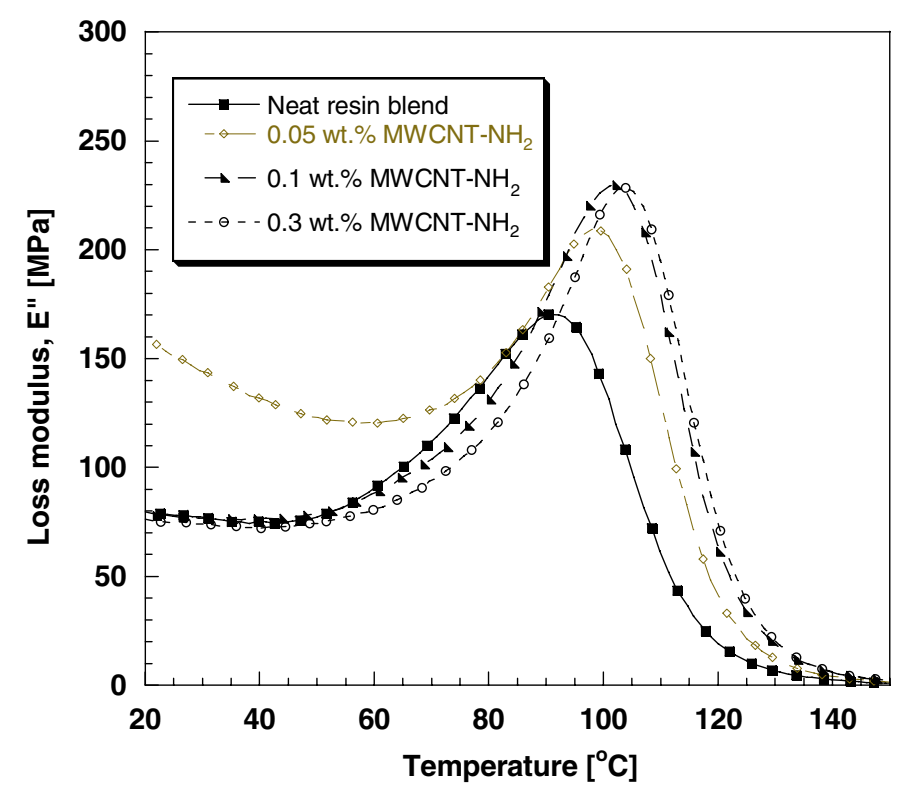

Fig. 10. Loss modulus values of nanocomposites containing amino-functionalized nanotubes (MWCNT- $\left.\mathrm{NH}_{2}\right)$.

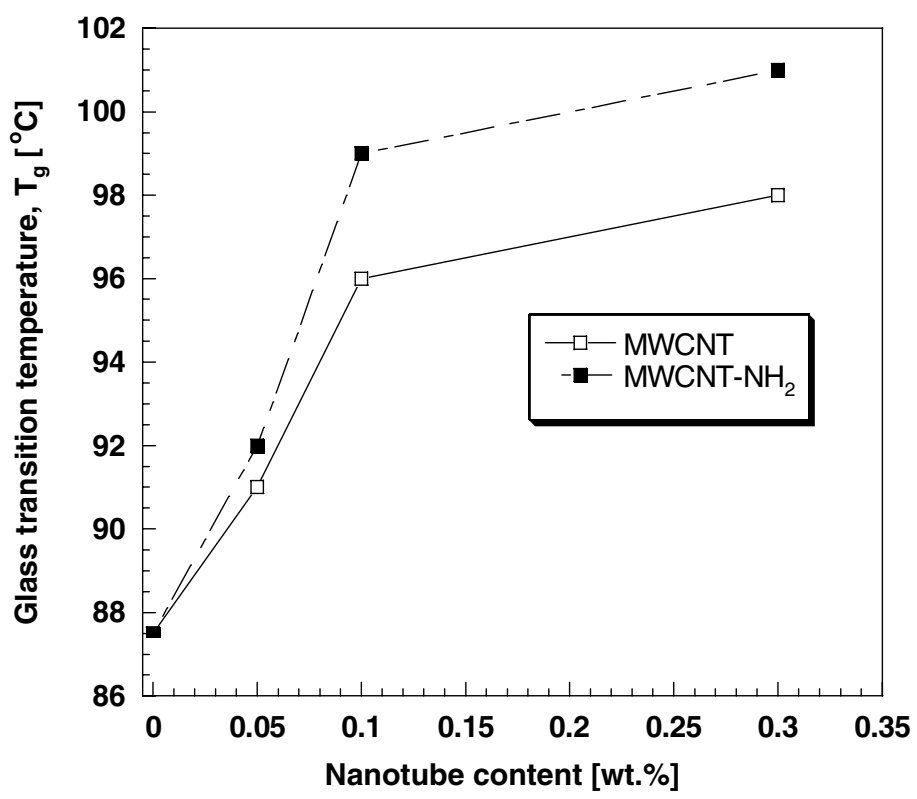

Fig. 11. Glass transition temperatures of the nanocomposites as a function of nanotube content with and without any functional groups.

nanotubes, thus a enhanced bonding at the interface further increases the $T_{\mathrm{g}}$. In addition, the reduced aspect ratio of amino-functionalized nanotubes may also have some effects on the degree of polymerization. Gryschuk et al. [13] stated that the lower aspect ratio of MWCNTs (achieved by ball milling process) would give better results for highly cross-linked thermoset resins such as vinyl-esters.

\subsection{Dispersion state of carbon nanotubes within the nanocomposites}

Figs. 12-a and 12-b are the TEM micrographs showing achieved dispersion state of MWCNT and MWCNT- $\mathrm{NH}_{2}$ at $0.3 \mathrm{wt} . \%$ loading within the corresponding resin systems, respectively. Functionalized nanotubes exhibited relatively good 


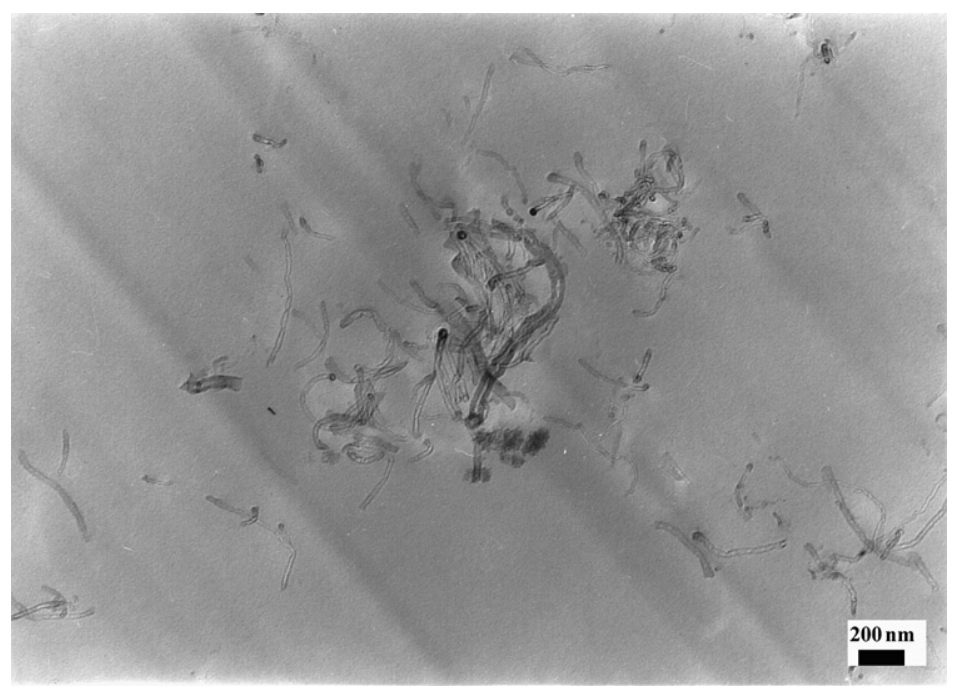

Fig. 12-a. TEM image showing the dispersion state of MWCNTs $(0.3 \mathrm{wt} . \%)$ within the vinyl ester/polyester resin matrix. No condensed agglomerates, but individual nanotubes are noticeable.

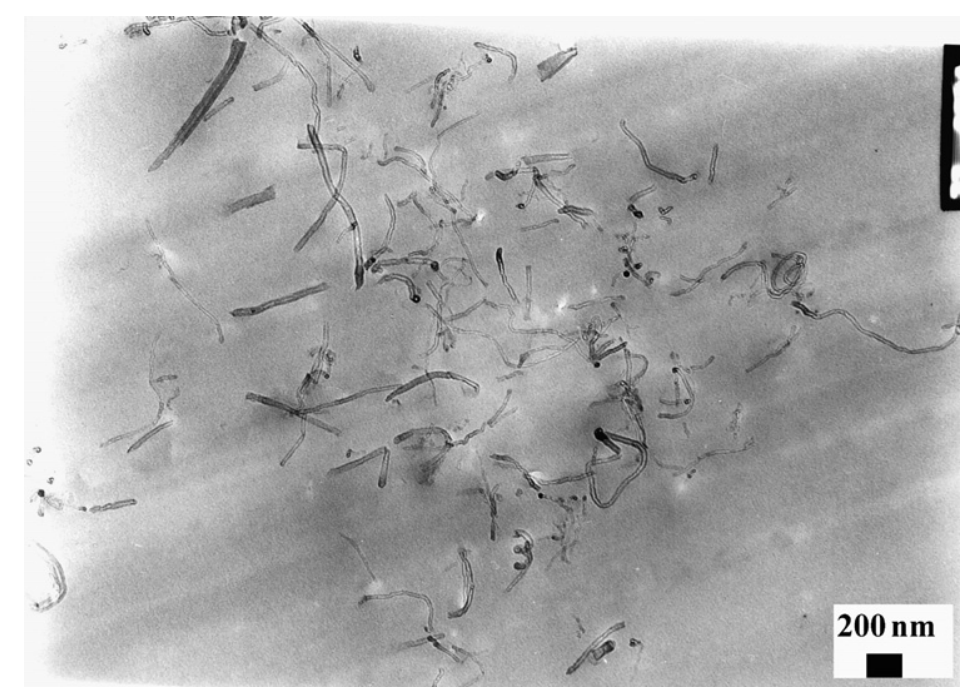

Fig. 12-b. TEM image showing the dispersion state of amino-functionalized multi walled nanotubes $\left(\mathrm{MWCNT}-\mathrm{NH}_{2}\right)(0.3 \mathrm{wt} . \%)$ within the corresponding vinyl ester/polyester resin matrix. A better dispersion of functionalized nanotubes within the resin is visible.

dispersion within the matrix resin blend without any significant agglomerates. The TEM characterizations also support our findings that amine functional groups over CNTs improve their dispersion state within the resins enhancing their compatibility with resin blend matrix.

\section{Conclusions}

In this study, the rheological behavior of the vinyl ester/polyester resin blend suspensions containing different weight percentage of MWCNTs and MWCNT- $\mathrm{NH}_{2}$ was investigated. CNTs with and without amine groups were dispersed within the corresponding resin blend using 3-roll milling method. These resin suspensions were then polymerized to reveal the effects of amine functional groups over the CNTs surfaces on the chemical interactions at the interface. The interactions were monitored by considering the rheological behavior (liquid state) in comparison with the properties of their matching nanocomposites (solid state). In that manner, dynamic mechanical behavior of the resulting nanocomposites was also investigated. Linear dynamic 
viscoelastic measurements revealed that storage modulus $\left(G^{\prime}\right)$ and loss modulus $\left(G^{\prime \prime}\right)$ values of resin suspensions as a function of angular frequency increased with respect to CNTs regardless of amine functional groups. Moreover, as a consequence of steady shear viscosity measurements, it was observed that each resin suspension containing nanotubes exhibited shear thinning behavior, while the neat resin blend was almost the Newtonian fluid. On the other hand, the storage $\left(E^{\prime}\right)$ and loss modulus $\left(E^{\prime \prime}\right)$ values of the resulting nanocomposites were found to increase with an increase in contents of CNTs. In addition, at $0.3 \mathrm{wt} . \%$ loading rate, resin suspensions showed pseudo solid like behavior at lower frequency. Furthermore, the incorporation of nanotubes into the resin blend shifted the corresponding $T_{\mathrm{g}}$ of the nanocomposites to higher values as compared to those of neat resin blend due to the reduced mobility of the surrounding resin blend matrix by CNTs. Furthermore, TEM was conducted to highlight the dispersion state of CNTs with and without amine functional groups within the cured resin samples. It was found that CNTs were dispersed adequately within the resin blend, which also proved that 3-roll milling technique was capable of providing homogenous dispersion of nano-fillers within thermoset resins to manufacture nanocomposites.

In brief, we found similar rheological properties from resin suspensions independent of amine groups. We also obtained relatively higher storage and loss modulus values as well as higher glass transition temperatures from the nanocomposites containing MWCNT- $\mathrm{NH}_{2}$. This is the corroborating evidence that enhanced dispersion state of aminofunctionalized nanotubes within the resin blend matrix compensates their lower aspect ratio promoting the chemical interactions at the interface between CNTs and resin blend matrix.

\section{Acknowledgements}

Authors acknowledge the supports of The Scientific and Technical Research Council of Turkey
(TÜBITAK) and JULİCH Research Center of Germany for the financial support for Tubitak-Julich 5 project.

\section{References}

[1] Gojny FH, Schulte K. Functionalization effect on the thermo-mechanical behavior of multiwalled carbon nanotube/epoxy composites. Compos Sci Technol 2004;34: 2303-8.

[2] Gojny FH, Wichmann M, Fiedler B, Schulte K. Influence of different carbon nanotubes on the mechanical properties of epoxy matrix composites-a comparative study. Compos Sci Technol 2005;65:2300-13.

[3] Gojny FH, Nastalczyk J, Roslaniec Z, Schulte K. Surface modified multiwalled carbon nanotubes in CNT/epoxy composites. Chem Phys Lett 2003;370:820-4.

[4] Gojny FH, Wichmann M, Köpke U, Fiedler B, Schulte K. Carbon nanotube-reinforced epoxy composites: enhanced stiffness and fracture toughness at low nanotube content. Compos Sci Technol 2004;34:2368-71.

[5] Sandler J, Schaffer MSP, Prase T, Bauhofer W, Schulte K, Windle AH, et al. Polymer 1999;40:5967-71.

[6] Pötsche P, Battachyya AR, Janke A. Melt mixing of polycarbonate with multiwalled carbon nanotubes: microscopic studies on the sate of dispersion. Eur Polym J 2004;40:137-48.

[7] Kinloch IA, Roberts SA, Windle AH. A rheological study of concentrated aqueous nanotube dispersions. Polymer 2002; 43:7483-91.

[8] Kim JA, Seong DG, Kang TJ, Youn JR. Effects of surface modification on rheological and mechanical properties of CNT/epoxy composites. Carbon 2006;44:1898-905.

[9] Song YS, Youn JR. Influence of dispersion sates of carbon nanotubes on physical properties of epoxy nanocomposites. Carbon 2005;43:1378-85.

[10] Mitchell CA, Bahr JL, Arepalli S, Tour JM. Dispersion of functionalized carbon nanotubes in polystyrene. Macromolecules 2002;35:8825-30.

[11] Amari T, Uesugi K, Suzuki H. Viscoelastic properties of carbon black suspension as a flocculated percolation system. Prog Org Coat 1997;31:171-8.

[12] Fidelus JD, Wiesel E, Gojny FH, Schulte K, Wagner HD. Thermo-mechanical properties of randomly oriented carbon/epoxy nanocomposites. Compos Part A 2005;36: $1555-1561$.

[13] Gryschuck O, Kocsis JK, Thomann R, Kanya Z, Kiricsi I. Multiwall carbon nanotube modified vinyl ester and vinyl ester - based hybrid resins. Compos Part: A 2006;37: 1252-9. 